\title{
Elaboração de cartilha de orientação para uso de telemetria cardíaca
}

\author{
Preparation of an orientation booklet for the use of cardiac telemetry \\ Elaboración de una guía orientativa para el uso de telemetría cardíaca
}

Rozemy Magda Vieira Gonçalves ${ }^{1 *}$, João Lucas Campos de Oliveira², Elizabete Rosane Palharini Yoneda Kahl' ${ }^{1}$, Mari Angela Victoria Lourenci Alves ${ }^{1}$.

\section{RESUMO}

Objetivo: Descrever a elaboração de uma cartilha de orientação para pacientes, profissionais e estudantes quanto ao uso da telemetria cardíaca. Relato de experiência: A cartilha de orientação foi elaborada a partir da vivência de enfermeiras hospitalares atuantes em uma unidade de cuidados especializados que recebe pacientes para o uso dessa tecnologia, localizada no sul do Brasil. Além disso, o devido levantamento técnico-científico associado às necessidades educativas percebidas para os pacientes, familiares, docentes, discentes e quadro funcional quanto ao uso da telemetria cardíaca foram respeitados. A cartilha ficou composta por: capa/contracapa; apresentação; sumário; lista de siglas; conceito de telemetria; 0 funcionamento da telemetria; indicações para o uso da telemetria; os benefícios ao utilizar essa tecnologia do cuidado; o uso correto dos eletrodos; cuidados para a manutenção do equipamento; o que o paciente pode fazer usando a telemetria e os motivos pelos quais é retirada a telemetria. Considerações finais: Acredita-se que a cartilha pode auxiliar na promoção do tratamento, especialmente nas condições que envolvem alterações no ritmo cardíaco, prevenindo possíveis complicações. Outros níveis de validação do material são recomendados.

Palavras-chave: Telemetria, Enfermagem cardiovascular, Cuidados de enfermagem, Educação em enfermagem, Autocuidado.

\begin{abstract}
Objective: To describe the development of a guidebook for patients, professionals and students regarding the use of cardiac telemetry. Experience report: The guidance booklet was created from the experience of hospital nurses working in a specialized care unit that receives patients for the use of this technology, located in southern Brazil. In addition, the proper technical-scientific research data associated with the perceived educational needs perceived by patients, family members, teachers, students and staff regarding the use of cardiac telemetry were respected. The booklet was comprised of: cover/back cover; presentation; summary; list of acronyms; telemetry concept; how the telemetry works; indications for the use of telemetry; the benefits of using this care technology; the correct use of the electrodes; care for equipment maintenance; what the patient can do while using the telemetry; and why the telemetry is removed. Final considerations: It is believed that the booklet can help promote treatment, especially for conditions that involve changes in heart rhythm while also preventing possible complications. Other material validation levels are recommended.
\end{abstract}

Keywords: Telemetry, Cardiovascular nursing, Nursing care, Nursing education, Self-care.

\section{RESUMEN}

Objetivo: Describir la elaboración de una guía orientativa para pacientes, profesionales y estudiantes sobre el uso de la telemetría cardíaca. Informe de experiencia: La guía orientativa fue elaborada a partir de la experiencia vivida por enfermeras hospitalarias que trabajan en una unidad de cuidados especiales cuyos pacientes requieren el uso de esta tecnología, ubicada en el sur de Brasil. Además, se cumplió con la debida encuesta técnico-científica relativa a las necesidades educativas percibidas destinada a los pacientes, las familias, los profesores, los estudiantes y el personal vinculados con el uso de la telemetría cardíaca. La guía estaba compuesta por: portada/contraportada; presentación; resumen; lista de siglas;

\footnotetext{
${ }^{1}$ Hospital de Clínicas de Porto Alegre, Porto Alegre - RS. *E-mail: rozygong@gmail.com

2 Universidade Federal do Rio Grande do Sul (UFRGS), Porto Alegre - RS.
} 
concepto de telemetría; funcionamiento de la telemetría; indicaciones para el uso de la telemetría; beneficios de uso de esta tecnología de cuidados; uso correcto de los electrodos; cuidados para el mantenimiento del equipo; qué puede hacer el paciente con la telemetría y las razones por las que se retira la telemetría. Consideraciones finales: Se cree que la guía puede ayudar a promover el tratamiento, especialmente para las enfermedades que conllevan cambios del ritmo cardíaco, y prevenir posibles complicaciones. Se recomiendan otros niveles de validación del material.

Palabras clave: Telemetría, Enfermería cardiovascular, Atención de enfermería, Educación em enfermeira, Autocuidado.

\section{INTRODUÇÃO}

As doenças do aparelho circulatório têm sido a principal causa de morte na população mundial. Há predominância de condições cardiovasculares no sexo masculino e entre maiores de 60 anos, contudo, a taxa de mortalidade pode ser maior entre as mulheres. Estudos apontam a diminuição do número de internações relacionadas à insuficiência cardíaca (IC) no Brasil, o aumento da taxa de permanência hospitalar e da mortalidade intra-hospitalar, bem como a elevação de custos, a qual representou $132,8 \%$ a mais nas autorizações de internação hospitalar no Sistema Único de Saúde (SUS) em uma análise de 11 anos (MOTA MS, et al., 2020; KAUFMAN R, et al., 2015; FERNANDES ADF, et al., 2020).

A IC permanece sendo um problema de saúde pública no Brasil, já que é prevalente e seu aumento está relacionado ao envelhecimento da população e ao melhor manejo dos seus fatores de risco. Além disso, percebe-se a evolução no tratamento de doenças cardíacas no quesito medicações; a educação do paciente, o que favorece sua adesão ao tratamento; e uso de tecnologias que auxiliam o controle da doença e sobrevida. Entretanto, paralelo a isso, a complexidade dos pacientes ao longo do tempo é maior (KAUFMAN R, et al., 2015).

$\mathrm{Na}$ IC e em outras condições que envolvem deficiências no ritmo cardíaco, o monitoramento por telemetria dos pacientes internados em enfermaria é um exemplo do uso de tecnologia em prol do cuidado, favorecendo a resolução quando na ocorrência deste problema. A telemetria cardíaca é uma tecnologia de transmissão por rádio frequência que envia em tempo fidedigno e contínuo os sinais eletrocardiográficos de pacientes internados em enfermarias não críticas, através de uma rede wireless bluetooth para uma ampla tela de monitor que centraliza todos os pacientes em telemetria da instituição, situado em uma unidade de tratamento intensivo coronariana (GONÇALVES RMV, et al.,2021; LAUER RD, 2021). Assim, a telemetria pode ser definida como um sistema de transmissão remoto e de forma individualizada que possibilita um maior controle dos indicadores de desconformidades cardíacas (PÉREZ-RODRIGUES G, et al., 2015).

Pacientes com as mais diversas doenças cardíacas se beneficiam da telemetria, especialmente pela ausência da necessidade de internação em leitos de tratamento intensivo. Pacientes em pré-cirurgias ou pré-transplantes cardíacos, em uso de drogas inotrópicas que alteram o débito cardíaco e as mais diferentes doenças que provocam arritmias cardíacas também se beneficiam do uso da telemetria (GONÇALVES RMV, et al., 2021).

Com a monitorização eletrocardiográfica à distância e sincronizada com a unidade coronariana, os pacientes ao apresentarem qualquer sinal de alerta são prontamente atendidos, uma vez que as equipes da unidade coronariana são especializadas em eletrocardiograma (ECG) e arritmias. Portanto, atuam imediatamente acionando as enfermeiras da unidade não especializada ou comparecendo ao setor. Isso contribui para a detecção precoce de uma injúria cardíaca com ações e intervenções rápidas e eficazes para o restabelecimento do paciente (GONÇALVES RMV, et al., 2021).

As orientações quanto ao uso da telemetria são importantes para a adequada instalação e funcionamento dessa tecnologia de cuidado. Além disso, as orientações são importantes para a implementação de estratégias de enfrentamentos, pois, ao serem fornecidas aos pacientes, familiares e quadro funcional, se estabelece relação de confiança, auxiliando na manutenção do cuidado no enfrentamento da condição em que se apresenta (PACZEK RS, et al., 2021). 
Ante o exposto, considera-se relevante a elaboração de uma cartilha de orientações para auxiliar pacientes, familiares e equipes de saúde para o correto uso e manutenção do funcionamento da telemetria. Isso porque, os materiais informativos são úteis e práticos para divulgar informações aos profissionais de saúde e aos próprios pacientes. Tais materiais servem como uma estratégia de prevenção, auxiliando na identificação precoce de complicações e no tratamento das morbidades e na prevenção das suas sequelas (SANTOS BD, et al.,2020). No caso da enfermagem, cumpre salientar que a elaboração de um material informativo pode integrar profissionais do campo assistencial, estudantes e docentes, articulando essas esferas interessadas na melhoria do cuidado (XIMENES MA, et al., 2019).

$\mathrm{Na}$ área de enfermagem cardiovascular, em especial a respeito do uso de telemetria cardíaca, postula-se que ainda há incipiência de conhecimento no que diz respeito à construção de materiais capazes de auxiliar no processo de educação em saúde para os pacientes e de educação permanente para trabalhadores de enfermagem. Diante do que foi explanado, o objetivo deste estudo foi descrever a elaboração de uma cartilha de orientação para pacientes, profissionais e estudantes quanto ao uso da telemetria cardíaca.

\section{RELATO DE EXPERIÊNCIA}

Estudo descritivo, do tipo relato de experiência. O campo de estudo foi uma unidade de internação clínica que atende pacientes cardíacos e que dispõe da rede wireless para a conexão do sistema de telemetria. A unidade de internação está localizada em um hospital universitário de grande porte da região sul do país e que atende clientes pelo SUS. Este tipo de tecnologia começou a ser utilizada em 2017 e desde então aproximadamente 170 pacientes por ano são beneficiados (GONÇALVES RMV, et al., 2021).

Não houve a necessidade de aprovação pelo Comitê de Ética em Pesquisa (CEP), pois trata-se de um relato de experiência do qual os autores foram os sujeitos envolvidos na construção do material educativo. Não foi exposta a instituição ou as pessoas (pacientes, familiares e/ou outros profissionais que não os autores) neste processo. A pesquisa enquadra-se nos fundamentos de dispensa do CEP, especialmente por se tratar de estudo que tem por finalidade a inserção teórica de situações que surgem espontânea e eventualmente na prática profissional, desde que não despontem dados que possam identificar as pessoas envolvidas (MINISTÉRIO DA SAÚDE, 2016).

A cartilha foi elaborada a partir da vivência de enfermeiras da unidade de internação e do levantamento da necessidade de um material informativo e educativo para os pacientes, familiares, docentes, discentes e quadro funcional quanto ao uso dessa tecnologia de forma correta para auxiliar no tratamento dos pacientes que fazem uso da telemetria. Isso porque, mesmo sabendo que as informações orais são feitas, uma informação escrita reforça o que foi dito e auxilia o paciente a compreender que suas ações o beneficiam para uma adequada intervenção terapêutica (TORRES HC, et al., 2009) e os envolvidos tanto pacientes como a equipes de saúde podem sanar dúvidas de forma rápida e acessível.

Ao verificar os potenciais benefícios de uma informação escrita, foi construída a cartilha de orientação numa linguagem acessível e com ilustrações para uma melhor fixação visual e entendimento do leitor. É prudente destacar que, além da vivência clínica das enfermeiras da unidade, a devida busca e consulta à literatura científica pertinente e atual foi respeitada no processo de construção da cartilha. Entre os materiais de apoio, destacam-se os livros, panfletos, manuais, diretrizes da Sociedade Brasileira de Cardiologia (SBC), resumos, artigos e demais publicações científicas pertinentes e atualizadas sobre o assunto.

Após discutir o que contemplaria a escrita da cartilha, fez-se um sumário e a partir dele foram sendo desmembrados os assuntos. Inicialmente, abordou-se o tema "o que é telemetria", e logo na sequência apresenta-se uma figura que ilustra o aparelho. Adiante, informa-se sobre o funcionamento da telemetria, salientando que o paciente permanece em uma unidade de internação comum, mas está permanentemente monitorizado, e seu traçado eletrocardiográfico e a frequência cardíaca são acompanhados em um monitor na unidade coronariana. Com isso, explica-se também sobre o fluxo de atendimento em caso de necessidade.

Após explanar sobre as indicações para que o paciente utilize a telemetria e os benefícios de fazer o uso dessa tecnologia do cuidado, aborda-se sobre o uso correto dos eletrodos para obtenção de um traçado 
cardíaco correto. Para isso, são apresentadas imagens reforçando a adequada colocação, localização e memorização, inclusive por cores que os diferenciam.

Na sequência, a cartilha descreve os cuidados para a manutenção do equipamento, com destaque para atribuições da equipe de enfermagem e do próprio paciente ou acompanhnate. Lista-se o que o paciente pode fazer usando a telemetria, estando internado na unidade de internação. Finaliza-se exibindo os motivos da retirada total do sistema de telemetria. A Figura 1 ilustra o processo de construção da cartilha sobre o uso da telemetria cardíaca, até chegar à sua divulgação.

Figura 1 - Processo de construção e divulgação sobre o uso da telemetria cardíaca.

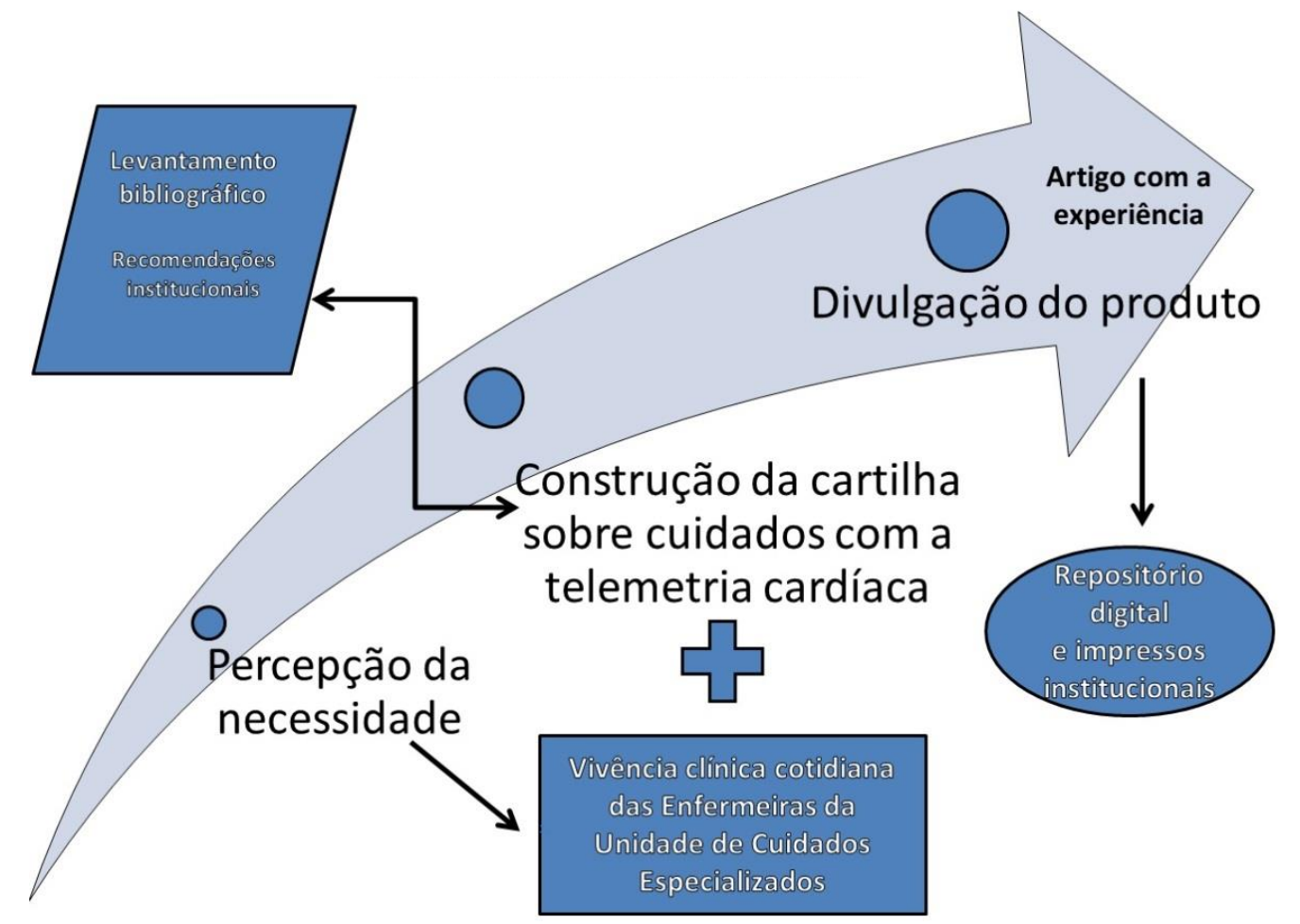

Fonte: Gonçalves RMV, et al., 2021.

A "Cartilha de Orientação para o uso de Telemetria Cardíaca" está disponível online em um repositório digital institucional (GONÇALVES RMV, et al., 2021). Os leitores interessados podem, ainda, requerer aos autores do presente estudo o material na íntegra, salvaguardando a devida citação do produto e também deste artigo que trata do seu processo de construção.

\section{DISCUSSÃO}

A IC e as arritmias são doenças que tendem a ascender, devido à evolução de tratamentos e envelhecimento da população. As arritmias podem potencializar a ocorrência de internação prolongadas e, ou complicações, readmissão hospitalar em quadros de pós-operatório, assim como de acidente vascular encefálico, IC, quedas e demência (SZPALHER AS e BATALHA MC, 2019).

As internações por IC descompensada representam parcela de custo importante à saúde pública. Os principais fatores desencadeantes da descompensação por IC são a má adesão medicamentosa e os casos de infecção. As arritmias muitas vezes ocorrem paralelamente a essa patologia, estando presente como comorbidade (SILVA WT et al., 2020). Considerando estes pressupostos, justifica-se a construção da cartilha divulgada.

As arritmias são situações complexas, podem ocorrerem de forma assintomática ou subjetiva. As sintomatologias são diferentes de uma pessoa para outra. Podem ser detectadas por meio de distúrbios de condução, bloqueio atrioventricular, alteração da frequência cardíaca (bradicardia ou taquicardia), fibrilação 
atrial ou ventricular e palpitações. Os sintomas variam desde tontura, vertigem, náusea, precordialgia, dispneia, pré síncope, até parada cardíaca ou morte súbita (SZPALHER AS e BATALHA MC, 2019). Neste contexto, a importância do monitoramento via telemetria vem à tona, trazendo a enfermagem como protagonista na atuação deste processo de monitoração cardíaca e manejo de situações potencialmente críticas.

O uso de material informativo melhora a adesão dos pacientes aos mais diversos tipos de tratamento. As informações adicionais intensificam as informações verbais. As pessoas memorizam $20 \%$ do que lhes é verbalizado, $30 \%$ do que é visualizado e $50 \%$ do que é verbalizado e visualizado. As combinações entre o escutar, visualizar e falar chegam a $70 \%$ de aprendizado, e quando se acrescenta o fazer, a taxa alcança para 90\% (GOMES B e MARTINS SS, 2016; PACZEK RS, et al., 2021). Essa alusão remete a uma potencial intervenção de enfermagem a ser planejada pela construção da cartilha, qual seja: após prover o material ao paciente, o enfermeiro pode, em conjunto, proceder a elementos de cuidado para manutenção da tecnologia, por exemplo, a fim de garantir a efetividade e apreensão deste processo.

As tecnologias na saúde estão cada vez mais presentes no cotidiano das mais diversas profissões. A enfermagem é uma das profissões da área da saúde que está em constante aprendizado e a construção de uma cartilha online reforça a utilização e acessibilidade dos recursos tecnológicos para a educação e o despertar do conhecimento da própria equipe e pacientes (REIS DLA, et al., 2021). Além disso, os materiais informativos permitem a organização e a conformidade das informações, sendo muito utilizados nas instituições de saúde, disseminando a aprendizagem e possibilitando o conhecimento ao alcance de todos (COSTA CIA, et al., 2018).

A telemetria é uma tecnologia do cuidado e as orientações, ao serem disponibilizadas também via redes tecnológicas, aguçam o saber e torna possível a consulta para a retirada de dúvidas de forma ágil e prática. A confecção da cartilha em torno dessa tecnologia teve o propósito de informar os profissionais de saúde, pacientes e estudantes quanto ao uso correto da telemetria cardíaca. Estudos mostram que uma cartilha bem estruturada, com informações adequadas, linguagem acessível às pessoas que interessam, contribui para 0 alcance de resultados positivos para 0 autocuidado e, consequentemente, o apropriado funcionamento da tecnologia a ser utilizada (XIMENES MA, et al.,2019; BANDEIRA LR, et al.,2020, PAZECK RS, et al., 2021).

A equipe de enfermagem é responsável pelo cuidado de pacientes que internam nas enfermarias que dispõem de rede wireless para o uso da telemetria. Entre as intervenções de enfermagem para o paciente em uso dessa tecnologia, pode-se citar, primeiramente, a orientação do paciente e familiar sobre a indicação do seu uso e como é o funcionamento da transmissão dos dados para a unidade de cuidados coronarianos. Destaca-se, pela prática clínica, que é a equipe de enfermagem quem instala a telemetria no paciente, fixa os eletrodos nos locais específicos do tórax, conectando-os ao aparelho de telemetria. Devese estar atento à qualidade e ao devido posicionamento dos eletrodos no paciente, além de manter o alarme do aparelho ligado, para nos alertar quando ocorrer qualquer alteração no traçado eletrocardiográfico.

O enfermeiro é responsável pelo monitoramento do funcionamento da telemetria. Para isso, o paciente deve ser orientando sobre as atividades que pode realizar com o aparelho em bateria e na bolsa específica, como deambular, ir até o banheiro, tomar banho, assim como, quando estiver no leito, deixar o aparelho conectado a sua base que está conectada à rede elétrica, a fim de manter o carregamento da bateria. $\mathrm{A}$ manutenção da integridade do aparelho, assim como de sua limpeza, é de responsabilidade dos profissionais de saúde que $o$ atendem. Orientar que não se ausente da unidade de internação específica, a fim de manter o monitoramento contínuo de seu traçado de eletrocardiograma, já que, toda vez que se ausentar dali, para realização de exames, por exemplo, a unidade de cuidados coronarianos deve ser comunicada, assim como quando de seu retorno (GONÇALVES RMV, et al., 2021; LAUER RD, 2021).

Durante a elaboração da cartilha, notou-se que a educação em saúde é extremamente importante para a efetividade do tratamento dos pacientes. Portanto, é aconselhável que profissionais de saúde e líderes locais que, a partir do levantamento das necessidades das especialidades de saúde e contexto socioeconômico da clientela em que convivem e de seus conhecimentos prévios, viabilizem ambientes de ensino-aprendizagem de forma significativa (ALAGOAS, 2018). 
No contexto da assistência ao paciente cardíaco, a construção e viabilização efetiva de materiais educativos permeiam uma necessidade de cuidado, uma vez que as doenças do sistema cardiovascular são comumente de ordem crônica, o que demanda que o paciente tenha bastante conhecimento, autonomia e adesão às boas práticas de autocuidado (GUERREIRO MARJ, et al., 2020; OLIVEIRA APD, et al., 2020).

Vale destacar que é recomendado durante a avaliação de materiais educativos, que o público-alvo possa opinar sobre conteúdo, linguagem e aparência do material, para que seja adequado à população a qual se destina (XIMENES MA, et al., 2019).

Neste sentido, considera-se que a avaliação da equipe de enfermagem foi respeitada na construção da cartilha, no entanto, a validação (de aparência e conteúdo, por exemplo) por pacientes e familiares pode ser necessária, mesmo que na prática clínica as dúvidas destes sujeitos pelo uso da cartilha construída tenham sido consideradas como passíveis de resolução. Percebeu-se ao elaborar a cartilha que a educação em saúde se faz extremamente valiosa para a promoção do autocuidado aos pacientes e a instigação de saberes na equipe assistencial. As matérias educativas não substituem as orientações faladas, mas fomentam a prevenção de complicações em saúde e a coletivização de conhecimentos.

\section{REFERÊNCIAS}

1. ALAGOAS. Guia de Educação Permanente em Saúde (EPS). 2018. Disponível em: <http://www.educasesauead.saude.al.gov.br/pluginfile.php/1556/mod_resource/content/2/CARTILHA_EPS_ONLINE. pdf>. Acesso em: 10 jun. 2021.

2. BANDEIRA LR, et al. Atenção integral fragmentada a pessoa estomizada na rede de atenção à saúde. Escola Anna Nery, 2020; 24(3): e20190297.

3. MINISTÉRIO DA SAÚDE. Conselho Nacional de Saúde. Resolução no 510, de 7 de abril de 2016. Trata sobre as diretrizes e normas regulamentadoras de pesquisa em ciências humanas e sociais. Diário Oficial da União, Brasília, DF, 24 maio 2016.

4. COSTA CIA, et al. Construção e validação de materiais educativos para criança com doença crônica: uma revisão integrativa. Revista Enfermagem UERJ, 2018; 26: e34208.

5. FERNANDES ADF, et al. Insuficiência cardíaca no Brasil subdesenvolvido: análise de tendência de dez anos. Arquivos Brasileiros de Cardiologia, 2020; 114(2): 222-231.

6. GOMES B, MARTINS SS. Pessoa estomizada: análise das práticas educativas de enfermagem. Revista Estima, 2016; 14(3): 146-153.

7. GONÇALVES RMV, et al. Cartilha de orientação para o uso de telemetria cardíaca. Porto Alegre: UFRGS, 2021. Disponível em: <https://drive.google.com/file/d/1V7NwSXy8gR6x-xNWLMvMib4yfKPcXQnl/view?usp=drivesdk >. Acesso em: 20 ago. 2021.

8. GUERREIRO MARJ, et al. Telecuidado como uma estratégia de saúde para a adesão do paciente com insuficiência cardíaca - revisão integrativa. Revista Enfermería Global, 2020; 19(2): 608-624.

9. KAUFMAN R, et al. Insuficiência cardíaca: análise de 12 anos da evolução em internações hospitalares e mortalidade. Internacional Journal os Cardiovascular Sciences, 2015; 28(4): 276-281.

10. LAUER RD, et al. Cuidado seguro ao paciente com telemetria em unidade de cuidados especiais. Brazilian Journal of Health Review, 2021; 4(3): 9830-9835.

11. MOTA MS, et al. Perfil epidemiológico da mortalidade por doenças isquêmicas do coração. Brazilian Journal of Surgery and Clinical Research, 2020; 32(2): 7-10.

12. OLIVEIRA APD, et al. Educação em saúde: efetividade das intervenções em pacientes com insuficiência cardíaca. Revista Brasileira de Enfermagem, 2020; 73(2): e20180782.

13. PACZEK RS, et al. Elaboração de cartilha de orientação para pacientes com estomas de eliminação. Revista Eletrônica Acervo Saúde, 2021; 13(3): e7002.

14. PEREIRA MO, et al. Superando os desafios para oferecer formação de qualidade em enfermagem psiquiátrica. Revista Brasileira de Enfermagem, 2020; 73 (1): e20180208.

15. PÉREZ-RODRÍGUEZ G, et al. EL seguimiento telemétrico disminuye la visita a la sala de urgências y coste de atención em enfermos com insuficiência cardíacacrónica. Cirgía y Cirujanos. 2015; 83: 279---285.

16. REIS DLA, et al. Construção de Manual de Orientações para a Educação Permanente em Centro Cirúrgico: relato de experiência. Revista Eletrônica de Enfermagem, 2021; 13(3): e7002.

17. SANTOS BD, et al. Elaboração de materiais informativos escritos para divulgações e recomendações em fonoaudiologia para profissionais da saúde. Revista CEFAC, 2020; 22(4): e1520.

18. SILVA WT, et al. Características clínicas e comorbidades associadas à mortalidade por insuficiência cardíaca em um hospital de alta complexidade na Região Amazônica do Brasil. Revista Pan-Amazônica de Saúde. $2020 ; 11$.

19. SZPALHER AS, BATALHA MC. Arritmias cardíacas: Diagnósticos de Enfermagem baseados na Toxonomia da Nanda (2018-2020). Revista Eletrônica Acervo Saúde, 2019; 11(17): 1-9.

20. TORRES HC, et al. The process of creating guidebooks for orienting self-care in the Diabetes educational program. Revista Brasileira de Enfermagem, 2009; 62(2): 312-6.

21. XIMENES MA, et al. Construction and validation of educational booklet content for fall prevention in hospitals. Acta Paulista de Enfermagem, 2019; 32(4): 433-441. 